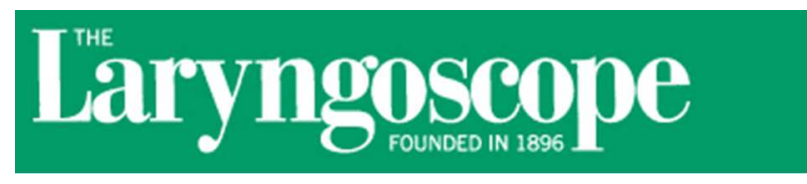

\title{
Transoral Endoscopic Nasopharyngectomy with a Next- Generation Flexible Robotic Surgical System
}

\begin{tabular}{|r|l|}
\hline Journal: & The Laryngoscope \\
\hline Manuscript ID & Iscope-15-2023.R1 \\
\hline Wiley - Manuscript type: & Original Reports \\
\hline Complete List of Authors: & $\begin{array}{l}\text { Tsang, Raymond; University of Hong Kong, Department of Surgery } \\
\text { Holsinger, Christopher; Stanford University, Department of Otolaryngology } \\
\text { - Head and Neck Surgery } \\
\text { Holsinger, Floyd; Stanford University, Otorhinolaryngology - Head and } \\
\text { Neck Surgery }\end{array}$ \\
\hline Keywords - Combo: & Head and Neck \\
\hline & \\
\hline
\end{tabular}

SCHOLARONE ${ }^{\text {m }}$

Manuscripts 


\title{
Transoral Endoscopic Nasopharyngectomy with A Flexible Next-
}

\author{
Generation Robotic Surgical System
}

Running title: Robotic Nasopharyngectomy With A Flexible Robot

Raymond K. Tsang, MS, FRCSEd(ORL) $)^{1}$

F. Christopher Holsinger MD, FACS ${ }^{2}$

${ }^{1}$ Division of Otorhinolaryngology - Head and Neck Surgery, Department of Surgery, University of Hong Kong.

${ }^{2}$ Division of Head and Neck Surgery, Department of Otolaryngology, Stanford University.

\section{Corresponding author:}

Floyd Christopher Holsinger, MD, FACS

Chief, Division Head and Neck Surgery $\square$

Director, Head and Neck Cancer/Thyroid Program

Professor of Otolaryngology

Stanford University,

875 Blake Wilbur Drive, $\square$

Palo Alto, CA 94305-5820

Tel: +1650.736 .1680$

Email: holsinger@stanford.edu

Conflict of interest: The authors declare no financial relationships relevant to the technology or companies utilized in this manuscript. According to Laryngoscope guidelines, this includes, but is not limited to, grants, employment, affiliations, patents, inventions, honoraria, consultancies, royalties, stock options/ownership, or expert testimony.

Level of Evidence: N/A, preclinical surgical research on cadavers.

Sources of Support: Intuitive Surgical, Inc. provided access to the da Vinci SP robotic surgical system and laboratory.

Keywords: Transoral robotic surgery, nasopharyngectomy, nasopharynx, nasopharyngeal carcinoma 


\begin{abstract}
Objective: To determine the feasibility of transoral endoscopic nasopharyngectomy without division of the soft plate using a flexible, next-generation robotic surgical system.
\end{abstract}

Study Design: Preclinical anatomic study using four human cadavers

Methods: Transoral resection of the nasopharyngeal wall with en-bloc resection of the cartilaginous Eustachian tube and dissection of the parapharyngeal fat space.

Results: The first flexible robotic surgical system has recently been described. We performed a series of laboratory experiments to determine whether this flexible system could be used to perform transoral robotic nasopharyngectomy. This novel system allowed docking of the patient-side cart at the side of the operating table. The cannula tip was placed approximately $12 \mathrm{~cm}$ from the edge of the retractor pointing superiorly towards the nasopharynx. Retraction of the soft palate anteriorly and tonsillar pillars laterally with "stay-sutures" expanded the velopharyngeal inlet, providing adequate space to deploy all four instruments (three surgical instruments and a camera) into the nasopharynx for dissection. All instruments could be deployed into the nasopharynx, without collision or restriction of joint movement in this cadaver model. Using this position and docking location, the new flexible surgical robot provided sufficient access, reach, and visualization to complete robotic nasopharyngectomy with en-bloc resection of the cartilaginous Eustachian tube. 
1

2

3

4

5

6

7

8

9

10

11

12

13

14

15

16

17

18

19

20

21

22

23

24

25

26

27

28

29

30

31

32

33

34

35

36

37

38

39

40

41

42

43

44

45

46

47

48

49

50

51

52

53

54

55

56

57

58

59

60

Conclusion: This feasibility study showed that transoral endoscopic nasopharyngectomy could be performed without compromising the integrity of the soft palate using a novel flexible robotic surgical system. 


\section{Introduction}

Over the past two decades, nasopharyngectomy has established its role in the salvage of recurrent or persistent carcinomas of the nasopharynx (NP) after radiation therapy. ${ }^{1}$ For small tumors with minimal lateral extension, endoscopic nasopharyngectomy has been shown to have good oncological results, and may be superior to re-irradiation. ${ }^{2}$ However, challenges remain with this surgical approach. The chief difficulty is the manipulation of instruments in the small confined space in the nasopharynx. However, there have been significant improvements and variety of new techniques in transoral endoscopic head and neck surgery (eHNS) that may promise new hope for patients faced with this disease. ${ }^{3}$

The da Vinci robotic surgical system was designed to assist surgeons in performing complex surgical maneuvers in small confined spaces of the human body. This system has been applied in the head and neck region for resection of oropharyngeal, laryngeal and hypopharyngeal tumors with a minimally invasive approach. Weinstein and O'Malley coined these operations as transoral robotic surgery (TORS) ${ }^{4}$. In December 2009, the FDA approved the use of the da Vinci surgical robotic (Intuitive Surgical Inc., Sunnyvale, CA) for resection of T1-2 cancers of the oropharynx, larynx and hypopharynx..$^{5}$

Ozer and Waltonen had already proposed the use of this system as part of transoral endoscopic nasopharyngectomy in a human cadaver model in $2008 .^{6}$ The Ohio State team divided the soft palate in the midline and retracted the two halves of the palate laterally, then deployed the surgical robot through the mouth to resect the lateral nasopharyngeal wall in their preclinical study. Meanwhile, working in Hong Kong, Wei and Ho described the first clinical case of transoral endoscopic 
nasopharyngectomy using robotic assistance and with a technique only slightly modified from that proposed at Ohio State. ${ }^{7}$

While these early experiences showed the potential of transoral eHNS using robotics for nasopharyngectomy, several hurdles remained. First, the size of the instruments of the current da Vinci Si surgical robotic system (Intuitive Surgical, Inc., Sunnyvale, CA) is larger than ideal for use in the head, neck and mouth. This mismatch of instrument size is even more prominent when deploying the robotic instruments from the mouth into the nasopharynx, which is an even smaller space and further confined by bony walls in all sides except the inferior wall. In order to deploy first-generation surgical robotic systems into the nasopharynx, normal structures such as the soft palate would need to be transgressed. However, the division of the soft palate and re-approximation at the conclusion of the procedure is not without risk: velopharyngeal insufficiency and wound healing after radiation therapy pose significant problems for patients and their surgeons. A robotic surgical system that is better suited to the head and neck might be able to be deployed in the nasopharynx without transgressing major structures, similar to a pure nasal endoscopic approach, and thus might be ideal for nasopharyngectomy.

In April 2014, the first-ever flexible robotic surgical system (Da Vinci® $\mathrm{SP}^{\mathrm{TM}}$ Surgical System, Model SP999, Endo Wrist ${ }^{\circledR} \mathrm{SP}^{\mathrm{TM}}$, Intuitive Surgical, Inc., Sunnyvale, CA) was approved by the Food and Drug Administration for use in genitourinary surgery ${ }^{8}$. This single-port surgical robot has been used as part of a human clinical trial in urological surgery, both prostatectomy and nephrectomy. ${ }^{9}$ This next-generation system has three flexible instruments and stereoscopic binocular camera, all confined in a cannula of $2.5-\mathrm{cm}$ diameter. These flexible robotic arms 
allow the instruments to be deployed from the oral cavity and turn upwards at the oropharynx towards the nasopharynx to perform surgical maneuvers. A detailed description of the design of the robot and its application in TORS has been published. ${ }^{10}$

Using a human cadaver model, we present the results of a preclinical study investigating the feasibility of adapting this flexible, next-generation surgical robot to perform nasopharyngectomy and dissection of the parapharyngeal space without dividing the soft palate.

\section{Material and Methods}

Four fresh cadavers with full dentition were used for dissection experiments. The dissections were performed in the Research and Design Laboratory of Intuitive Surgical, Inc., in Sunnyvale, CA. Intuitive Surgical Inc. provided cadavers, access to the da Vinci SP prototype robot and engineer support. The first cadaver was used as a pilot study and the soft palate was split early in the dissection. No measurements were made on this cadaver. On the subsequent three cadavers, the soft palate was retracted only with 2-0 silk "stay-sutures" and no incisions were made. Measurements recorded included the weight and height of the cadavers, inter-dental distance, size of the soft palate entrance after retraction and the distance of the lateral boundary of surgical defect from midline.

\section{$\underline{\text { Patient preparation }}$}

The cadavers were placed on the laboratory operating table without a shoulder roll. The table was tilted head up at an approximate angle of $10^{\circ}$. To simulate the actual operating room environment, the cadavers were intubated with a size 7 
endotracheal tube. A Dingman retractor was placed in to the oral cavity and was fixed to an endoscope holder (Karl Storz, Tuttlingen, Germany) which was secured to the operating table. A 2-0 silk stitch was placed through the uvula and distal soft palate to retract the soft palate anteriorly. 2-0 silk stitches were then passed from the anterior pillar of tonsil through the posterior pillar to retract laterally the whole tonsillar pillar on both sides. Figure 1 shows the view of the oral cavity after the placement of Dingman retractor and stay-sutures.

\section{Docking}

The flexible robotic surgical system has a single arm that can swivel more than $360^{\circ}$ on the boom. Thus, the patient cart can be docked from the patient's right side, left side or even from the head side of the patient. We elected to dock the cart from the left side of the patient. For comparison, the first generation surgical robot must be deployed and docked above the patient's head in order to approach the nasopharynx. Figure 2 shows the docking of the da Vinci SP and the docking of the da Vinci Si robot for comparison. From previous experience using the da Vinci SP robot to perform oropharyngectomy, a working distance of 10-12 cm away from the retractor was estimated. This configuration permitted the robotic instruments to be deployed past the cannula enabling full articulation. Therefore, the cannula was placed approximately $12 \mathrm{~cm}$ away from the Dingman retractor, at an angle parallel to the tongue blade of the Dingman retractor. The camera was placed in the lower quadrant of the cannula with the tip just inside the oral cavity. From the surgeon console, the surgeon controls the camera and navigates it into the operative position. To achieve optimal visualization, the endoscopic camera was first driven deep into the oropharynx while flexing the proximal joint of the camera slightly inferiorly. As the 
endoscopic camera approached the posterior oropharyngeal wall, the distal joint of the camera was then flexed upwards towards the nasopharynx to angle of approximately $30^{\circ}$. The nasopharynx came into view. From the console, the surgeon can then refine the view by retracting the endoscopic camera back. Figure 3 shows the view of the nasopharynx captured with the endoscopic camera of the Vinci SP robot.

Robotic instruments were then inserted. For right nasopharyngectomy, a monopolar cautery spatula was inserted on the left side of the cannula (left hand instrument) and bipolar Maryland forceps were inserted on the right side (right hand instrument). Conversely, the two instruments could be swapped for dissection of the left side. Alternatively, monopolar cautery scissors could be used instead of a monopolar cautery spatula. Using the flexible robotic system, a fourth instrument was then deployed for this transoral endoscopic resection of the nasopharynx: a fenestrated bipolar grasping forceps was inserted in the top quadrant of the cannula and was controlled by either the left or right hand. This grasping forceps was used to retract the soft palate superiorly to improve the exposure and later, to retract the specimen.

\section{$\underline{\text { Robotic Nasopharyngectomy }}$}

The following is a short description of a right nasopharyngectomy with enbloc resection of the right Eustachian tube using the da Vinci SP robot. First, margins around the resection were marked with a surgical marking pen at least 5-10mm away from the target anatomy to ensure an adequate resection. The anterior cushion of the right Eustachian tube was separated from the posterior choana. The pharyngobasilar fascia was also detached from the medial pterygoid plate. To dissect the lateral nasopharynx, the Maryland forceps grasped the Eustachian tube cushion and retracted 
it medially. The monopolar cautery was then used to divide the attachment of the tensor veli palatini muscle. The parapharyngeal fat was then dissected out until the origin of the medial pterygoid muscle was exposed (Figure 4). The nasopharynx mucosa was dissected with the Maryland forceps grasping the mucosa. The underlying prevertebral fascia could also be dissected en-bloc with the nasopharynx mucosa if necessary. The dissection of the nasopharynx mucosa converged at the Eustachian tube and finally the cartilaginous Eustachian tube was divided near the bony Eustachian tube. The specimen was then freed from surrounding attachments and delivered. During the latter part of the dissection, the bipolar fenestrated forceps was used to grasp the specimen and provide additional traction, freeing the Maryland forceps for dissection (Figure 5).

\section{Dissection of the parapharyngeal space}

For cadaver 2, after completing a right nasopharyngectomy, the contents of the parapharyngeal space adjacent to the nasopharynx were removed. The internal carotid artery was exposed after careful dissection of the carotid sheath. Subsequently, the origin of the medial pterygoid muscles was resected, exposing the lateral pterygoid muscle. The mandibular branch of the trigeminal nerve (V3) could be seen lying on the lateral pterygoid muscle. V3 was followed superiorly up to the foramen ovale. Figure 6 shows the view from the camera at the end of this extended dissection. The internal carotid artery and mandibular branch of trigeminal nerve had been dissected out. When dissecting in the deeper part of the nasopharynx and parapharyngeal space in order to get the desired view, instead of moving the endoscopic camera closer to the operating field, we applied 2x and 4x digital zoom for a magnified view. This allowed the camera to be positioned further away from the 
robotic instruments to avoid collisions. The resolution of the image after digital magnification provided adequate visualization for a meticulous dissection in this space.

\section{Results}

Table 1 shows the measurements of cadavers, the dimensions of the dissection cavity created by elevating the soft palate and the lateral reach of the instruments. For cadaver 3 , due the limited mouth opening $(45 \mathrm{~mm}$ compared to $50 \mathrm{~mm}$ and $55 \mathrm{~mm}$ of the other 2 cadavers), we were unable to view the posterior edge of the vomer. To improve the elevation of the soft palate, a small cut was made over the edge of the soft palate just above the upper pole of the tonsils on each side. This allowed further elevation of the soft palate enabling visualization of the posterior edge of the vomer.

We were able to perform robotic nasopharyngectomy with en-bloc resection of the cartilaginous Eustachian tube in all three cadavers. The cartilaginous Eustachian tube was divided at the junction with the bony Eustachian tube. Although 3 surgical instruments and a stereoscopic endoscope were deployed into the nasopharynx, there were no collisions between the robotic instruments. The use of four instruments within the NP using this flexible surgical robot represents a significant advance over first-generation robotic systems. There were no accidental or non-intentional injuries to the perioral tissue, tongue and soft palate. Video 1 demonstrates the technique of transoral endoscopic nasopharyngectomy using the flexible robotic system.

The single cannula and flexible instrument configuration of the da Vinci SP robot allowed lateral dissection in the parapharyngeal space via a transoral route without the need to divide the soft palate for access. The lateral reach of the 
instruments can extend beyond the lateral pterygoid plate into the masticator space as evident in the cadaver 2 .

\section{Discussion}

The da Vinci SP is a next-generation flexible surgical robot, with distinct potential advantages in the head and neck, especially in the nasopharynx and skull base. With three instruments and a stereoscopic endoscope camera all delivered through a single $2.5-\mathrm{cm}$ cannula, all four robotic instruments can be easily deployed into the mouth. The incorporation of a proximal joint or "elbow" in the robotic instruments allows three robotic arms to triangulate onto target surgical anatomy through a narrow aperture with a "working space" roughly the size of a tennis ball. In the previous generations of robot surgical systems, instrument triangulation is accomplished by moving the rigid arms outside the patient, increasing the chance of instrument collisions. In the work presented here, even with 3 robotic instruments deployed in the tight space of nasopharynx, there were no instrument collisions. The diameters of the robotic instruments are six millimeters with the same seven degrees of freedom and motion scaling as in previous generations of this technology. While there is no direct comparison on the radius of bending of the effector arm between the $5 \mathrm{~mm}$ da Vinci Si robot and the $6 \mathrm{~mm}$ da Vinci SP, the authors experiences with the da Vinci SP system are that the instrument reach and dexterity of da Vinci SP robotic instruments are at least on par if not better than the $5 \mathrm{~mm}$ da Vinci Si robotic instruments. The da Vinci SP also has motion scaling similar to the da Vinci Si machine, allowing precise dissection. 
The flexible camera is also an advantage. This feature facilitates nearly instantaneous adjustment of the stereoscopic endoscope without instrument change. Further, the flexible endoscopic camera can be bent into an S-shape and turn around corners for visualization. Thus, the angle of visualization during dissection might be precisely customized to future human patient's anatomy, rather than limited by zero or thirty degree endoscopes. Coupled with the digital zoom, the new flexible endoscopic camera system reduces the chance of collision with other robotic instruments.

The soft palate is the key obstacle in the transoral approach to the nasopharynx. Fee et al. described the transoral, transpalatal approach to the nasopharynx in $1988 .^{11}$ In this approach, the authors fashioned a posteriorly based palatal mucosal flap, detached the soft palate from the hard palate and removed the palatine bone for visualization and access. When Wei et al. described the approach for deploying radioactive gold grain to the nasopharynx for brachytherapy, the soft palate was split in the midline for access. ${ }^{12}$ The same split-palate approach was adopted by Ozer and Waltonen in 2008 for the cadaveric experiment on robotic nasopharyngectomy. Splitting the soft palate has distinct disadvantages, as there are risks, including palatal fistula and wound contracture of the scar. Both can lead to velopharyngeal incompetence.

For this reason, other experimental approaches for robotic assisted nasopharyngectomy have been described. Dallan et al. described placing the two robotic instruments through small incisions in the submandibular area on both sides and placing the telescope through the nasal cavity. ${ }^{13}$. The disadvantage of the submandibular port approach include inadvertent injuries to the marginal branch of 
the facial nerve, as well as other structures of the floor of mouth and risk of orocutaneous fistula. Cho et al. abandoned the transoral approach entirely and described an entirely transnasal approach to the nasopharynx. ${ }^{14}$ However, the approach required bilateral medial maxillectomies to increase the working space in the nasal cavities.

This new flexible robotic surgical system with endoscopic camera and three additional instruments allowed approaching the nasopharynx via the oral cavity without splitting the palate. This inferior approach offered space for robotic instrument movement and access to the parapharyngeal space. The dissection could be extended to the masticator space and in cadaver 2, the mandibular nerve was exposed after resection of the medial pterygoid muscle. An approach using a lateral palatal flap approach required division and retraction of the soft palate to dissect the parapharyngeal space and expose the mandibular nerve. ${ }^{15}$ Thus, the da Vinci SP system has a major advantage over first-generation of surgical robots and other nonrobotic systems by achieving the same instrument reach without major disruption of the integrity of the soft palate.

For instance, Richmon has described transoral endoscopic nasopharyngectomy using the "Flex System" in human cadavers. ${ }^{16}$ The Flex System (Medrobotics, Raynham, MA) employs laparoendoscopic instruments and a snakelike flexible camera for endoscopic surgery of the head and neck. Since the surgeon's hands manipulate two laparoscopic instruments to perform the surgery, this system provides haptic feedback and retains some tactile sensation, which may be of value to surgeons performing transoral endoscopic head and neck surgery.

A significant advantage of the da Vinci SP is the use of three instruments to perform transoral endoscopic nasopharyngectomy. The use of this third arm 
optimizes the surgeon's ability to provide traction and counter-traction in the confined space of the NP. By manipulating the specimen with this third arm, the surgeon can better dissect critical neurovascular structures and maintain a better view of the dissection, as evident in Figure 6. Previously this would require the bedside surgeon to insert a grasping forceps via the nostril to provide traction. Finally, the primary surgeon, rather than the bedside assistant, may elect to have control over traction and counter-traction, as well as other tasks such as providing suction of the operation field.

As previously described using the da Vinci SP for TORS lateral oropharyngectomy, ${ }^{10}$ the design of the patient cart with more than $360^{\circ}$ rotation of the instrument arm makes docking of the robot easier. The patient cart can be docked from either side of the patient or the head of the patient. Docking from the side has several advantages. When needed, there will be more room for the bedside surgeon to assist the operation when this is required for hemostasis. But perhaps most importantly, the side-dock position of da Vinci SP may make it possible to deploy and use surgical navigation system intraoperatively. To et al. has previously described the use of navigation in localizing the internal carotid artery in nasopharyngectomy via a mid-face degloving approach ${ }^{17}$ but access from the head of the bed is needed. The possibility of now using a surgical navigation system in nasopharyngectomy will improve the safety of the surgery, especially in localizing the internal carotid artery. Thus, the ergonomics of this new surgical system may facilitate the routine incorporation of surgical navigation into robotic head and neck surgery of the NP.

However, there are limitations with this new surgical robot. There are no instruments capable of dividing or removing bone. Tumors in the roof of the nasopharynx frequently involve the bone of the sphenoid floor and clivus. For 
resection that requires bone removal, the endoscopic surgeon must still resort to endoscopic burs and drills for bone removal, similar to previous description of combined endoscopic and robotic nasopharyngectomy. ${ }^{18,19}$ Thus further innovation and refinement of robotic surgical systems for surgery of the nasopharynx is needed.

\section{Conclusion}

Preclinical testing in human cadavers has shown that the da Vinci SP robot can be adapted to perform robotic nasopharyngectomy via a transoral approach without the need to completely divide the soft palate. With improvement in instrument design, this new surgical robot offers multiple advantages over the previous generation of surgical robot.

Acknowledgement: The authors would like to thank Paul Lilagan and Dr. Christie Draper of Intuitive Surgical Inc. for providing engineering support and advice on the manuscript. 


\section{References}

1. Wei WI, Chan JYW, Ng RWM, Ho WK. Surgical salvage of persistent or recurrent nasopharyngeal carcinoma with maxillary swing approach - Critical appraisal after 2 decades. Head Neck. 2011;33(7):969-975.

2. You R, Zou X, Hua Y-J, et al. Salvage endoscopic nasopharyngectomy is superior to intensity-modulated radiation therapy for local recurrence of selected T1-T3 nasopharyngeal carcinoma - A case-matched comparison. Radiother Oncol. 2015;115(3):399-406.

3. Holsinger FC, Ferris RL. Transoral Endoscopic Head and Neck Surgery and Its Role Within the Multidisciplinary Treatment Paradigm of Oropharynx Cancer: Robotics, Lasers, and Clinical Trials. Journal of Clinical Oncology. 2015;33(29):3285-3292.

4. Weinstein GS, O'Malley BW, Hockstein NG. Transoral robotic surgery: supraglottic laryngectomy in a canine model. Laryngoscope. 2005;115(7):1315-1319.

5. 501(K) Summary, Section III: Indications for Use for Intuitive Surgical Endoscopic Instrument Control System for Transoral Otolaryngology Procedures. The United States Food and Drug Administration; 2009.I

6. Ozer E, Waltonen J. Transoral robotic nasopharyngectomy: a novel approach for nasopharyngeal lesions. Laryngoscope. 2008;118(9):1613-1616.

7. Wei WI, Ho W-K. Transoral robotic resection of recurrent nasopharyngeal carcinoma. Laryngoscope. 2010;120(10):2011-2014.

8. 501(K) Summary: Da Vinci ${ }^{\circledR}$ Sp $^{\mathrm{TM}}$ Surgical System Model SP999. The United States Food and Drug Administration; 2014.

9. Kaouk JH, Haber G-P, Autorino R, et al. A novel robotic system for single-port urologic surgery: first clinical investigation. Eur Urol. 2014;66(6):1033-1043.

10. Holsinger FC. Single-Arm, Flexible Robotic Surgical System for Transoral Resection of the Tonsil, Lateral Pharyngeal Wall. Laryngoscope. September 
2015, Epub ahead of print.

11. Fee WE, Gilmer PA, Goffinet DR. Surgical management of recurrent nasopharyngeal carcinoma after radiation failure at the primary site. Laryngoscope. 1988;98(11):1220-1226.

12. Wei W, Sham J, Choy D, Ho C, Lam K. Split-palate approach for gold grain implantation in nasopharyngeal carcinoma. Arch Otolaryngol Head Neck Surg. 1990;116(5):578-582.

13. Dallan I, Castelnuovo P, Montevecchi F, et al. Combined transoral transnasal robotic-assisted nasopharyngectomy: a cadaveric feasibility study. Eur Arch Otorhinolaryngol. 2011;269(1):235-239.

14. Cho H-J, Kang JW, Min HJ, et al. Robotic nasopharyngectomy via combined endonasal and transantral port: a preliminary cadaveric study. Laryngoscope. 2015;125(8):1839-1843.

15. Tsang RK, Mohr C. Lateral palatal flap approach to the nasopharynx and parapharyngeal space for transoral robotic surgery: a cadaveric study. Journal of Robotic Surgery. 2013;7(2):119-123.

16. Richmon JD. Transoral palate-sparing nasopharyngectomy with the Flex ${ }^{\circledR}$ System: preclinical study. Laryngoscope. 2015;125(2):318-322.

17. To E, Yuen E, Tsang W, et al. The use of stereotactic navigation guidance in minimally invasive transnasal nasopharyngectomy: a comparison with the conventional open transfacial approach. Br J Radiol. 2002;75(892):345-350.

18. Yin Tsang RK, Ho W-K, Wei WI. Combined transnasal endoscopic and transoral robotic resection of recurrent nasopharyngeal carcinoma. Head Neck. 2012;34(8):1190-1193.

19. Tsang RKY, Ho WK, Chan JYW, Wei WI. Combined robotic and endoscopic nasopharyngectomy for recurrent nasopharyngeal carcinoma. Asian Rhinology Journal. 2013;1(1):61-64. 


\section{Figure Legends}

Figure 1. Photo showing the entrance to the nasopharynx through the oral cavity after retraction of the soft palate anteriorly and the tonsillar pillars laterally with stitches.

Figure 2. A. Docking of the da Vinci SP robot from the side of the patient. B. Docking of the da Vinci Si robot from the head of the patient.

Figure 3. Screen capture showing the view of the nasopharynx through the endoscopic camera flexing upwards to view the nasopharynx.

Figure 4. Screen capture showing the dissection of the right parapharyngeal fat space exposing the origin of the right medial pterygoid muscle. MT denotes medial pterygoid muscle; $\mathrm{P}$ denotes parapharyngeal space; E denotes Eustachian tube cushion.

Figure 5. Screen capture showing the retraction of the dissected specimen upwards with the fenestrated bipolar forceps in the $3^{\text {rd }}$ arm of the da Vinci SP robot, freeing the Maryland forceps in the $2^{\text {nd }}$ arm for dissection and counter-traction.

Figure 6. View of the right parapharyngeal space after removal of the contents of the parapharyngeal space and resection of the right medial pterygoid muscle. Black arrow points to the internal carotid artery. White arrow points to the mandibular branch of the trigeminal nerve.

Video 1. Video of nasopharyngectomy performed with the da Vinci SP robot on a cadaver. For brevity, this video is edited and presented with an accelerated frame rate. 


\begin{tabular}{|c|c|c|c|}
\hline Measurements & Cadaver 1 & Cadaver 2 & Cadaver 3 \\
\hline Height and weight & $163 \mathrm{~cm}, 39 \mathrm{~kg}$ & $173 \mathrm{~cm}, 59 \mathrm{~kg}$ & $163 \mathrm{~cm}, 70 \mathrm{~kg}$ \\
\hline Dental gap & $55 \mathrm{~mm}$ & $55 \mathrm{~mm}$ & $45 \mathrm{~cm}$ \\
\hline $\begin{array}{l}\text { Distance between uvula and posterior pharyngeal wall after retraction of soft } \\
\text { palate }\end{array}$ & $35 \mathrm{~mm}$ & $35 \mathrm{~mm}$ & $35 \mathrm{~mm}$ \\
\hline
\end{tabular}




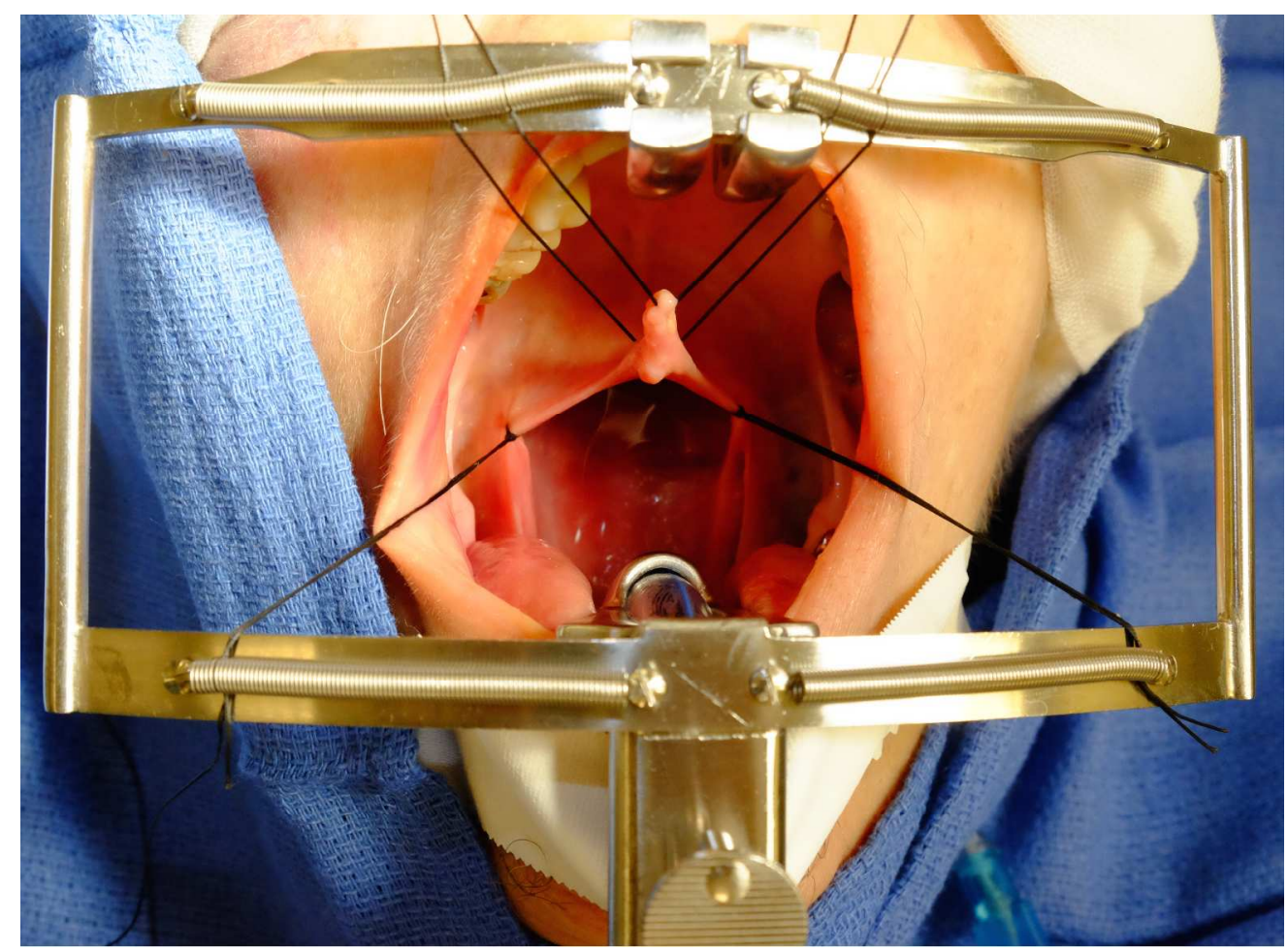

Figure 1. Photo showing the entrance to the nasopharynx through the oral cavity after retraction of the soft palate anteriorly and the tonsillar pillars laterally with stitches. $1555 \times 1148 \mathrm{~mm}(72 \times 72 \mathrm{DPI})$ 
Figure 2. A. Docking of the da Vinci SP robot from the side of the patient. B. Docking of the da Vinci Si robot from the head of the patient. $479 \times 388 \mathrm{~mm}(300 \times 300 \mathrm{DPI})$ 


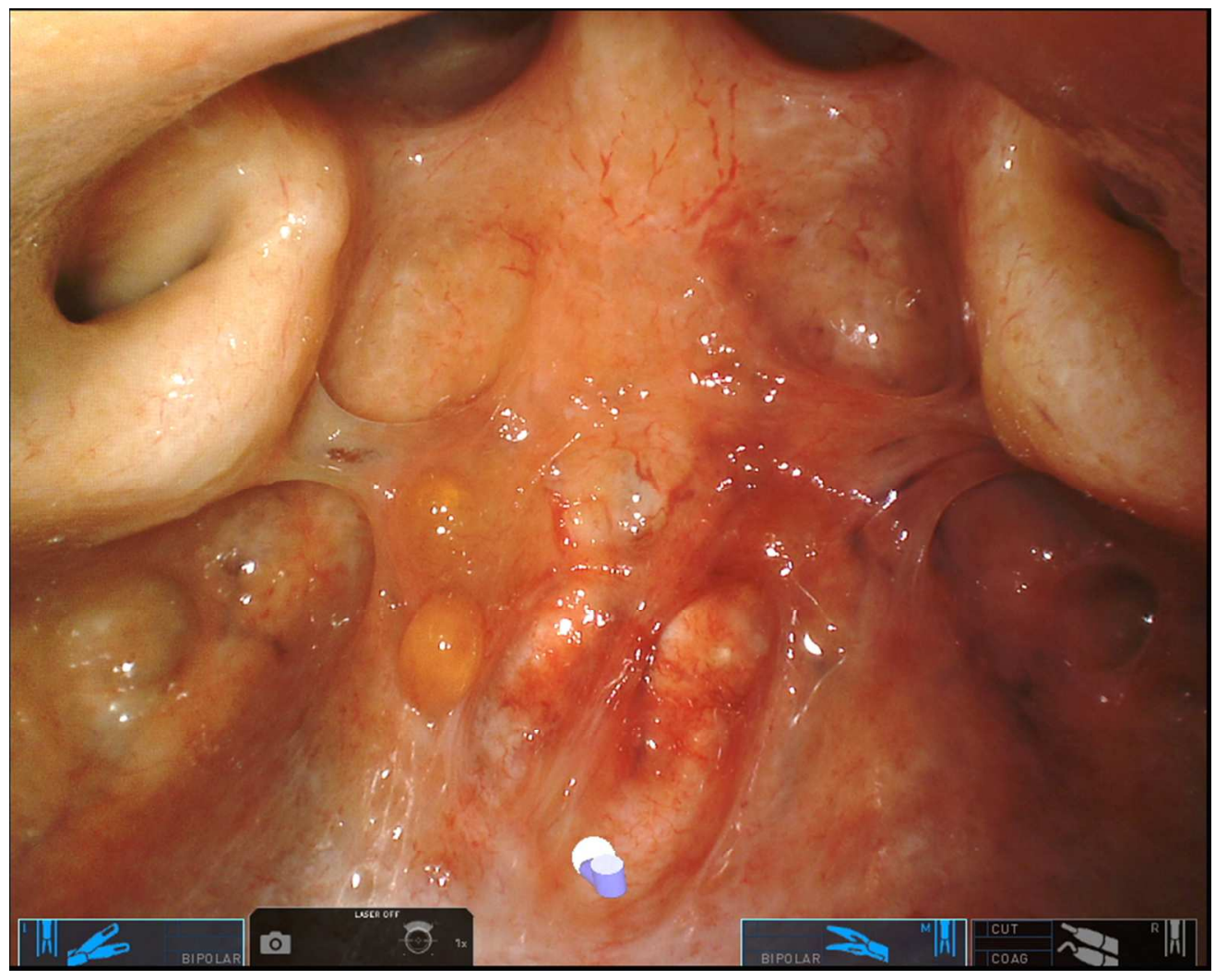

Figure 3. Screen capture showing the view of the nasopharynx through the endoscopic camera flexing upwards to view the nasopharynx. $316 \times 253 \mathrm{~mm}(72 \times 72 \mathrm{DPI})$ 
Figure 4. Screen capture showing the dissection of the right parapharyngeal fat space exposing the origin of the right medial pterygoid muscle. MT denotes medial pterygoid muscle; $\mathrm{P}$ denotes parapharyngeal space; $\mathrm{E}$ denotes Eustachian tube cushion $344 \times 253 \mathrm{~mm}(72 \times 72 \mathrm{DPI})$ 
Figure 5. Screen capture showing the retraction of the dissected specimen upwards with the fenestrated bipolar forceps in the 3rd arm of the da Vinci SP robot, freeing the Maryland forceps in the 2nd arm for dissection and counter-traction. $341 \times 252 \mathrm{~mm}(72 \times 72 \mathrm{DPI})$ 
Figure 6. View of the right parapharyngeal space after removal of the contents of the parapharyngeal space and resection of the right medial pterygoid muscle. Black arrow points to the internal carotid artery. White arrow points to the mandibular branch of the trigeminal nerve $225 \times 166 \mathrm{~mm}(72 \times 72 \mathrm{DPI})$ 